\title{
A hybrid queuing strategy for network traffic on scale-free networks
}

\author{
Kai-Quan $\mathrm{Cai}^{*}$ and $\mathrm{Lu} \mathrm{Yu}^{\dagger}$ \\ School of Electronic and Information Engineering, \\ Beihang University, Beijing 100191, China \\ National Key Laboratory of CNS/ATM, Beijing 100191, China \\ *caikq@buaa.edu.cn \\ †yulu73931@buaa.edu.cn \\ Yan-Bo Zhu ${ }^{\ddagger}$ \\ Aviation Data Communication Corporation, Beijing 100191, China \\ National Key Laboratory of CNS/ATM, Beijing 100191, China \\ yanbo_zhu@163.com
}

Received 1 December 2016

Accepted 25 January 2017

Published 15 February 2017

\begin{abstract}
In this paper, a hybrid queuing strategy (HQS) is proposed in traffic dynamics model on scale-free networks, where the delivery priority of packets in the queue is related to their distance to destination and the queue length of next jump. We compare the performance of the proposed HQS with that of the traditional first-in-first-out (FIFO) queuing strategy and the shortest-remaining-path-first (SRPF) queuing strategy proposed by Du et al. It is observed that the network traffic efficiency utilizing HQS with suitable value of parameter $h$ can be further improved in the congestion state. Our work provides new insights for the understanding of the networked-traffic systems.
\end{abstract}

Keywords: Scale-free network; traffic; queuing strategy.

\section{Introduction}

The problems of complex network, such as network robustness,,$\underline{1-3}$ evolutionary games,,$\frac{4,5}{}$ traffic dynamic, $\stackrel{6}{-9}$ optimization,,$\underline{10}-\underline{13}$ coupled networks ${ }^{14}$ and so on, have attracted a great deal of attention in the past decades due to the small-world

$\ddagger$ Corresponding author .

This is an Open Access article published by World Scientific Publishing Company. It is distributed under the terms of the Creative Commons Attribution 4.0 (CC-BY) License. Further distribution of this work is permitted, provided the original work is properly cited. 
phenomenon discovered by Watts and Strogatz, $\frac{15}{2}$ and the scale-free property by Barabási and Albert. ${ }^{16}$ As the traffic congestion becomes more and more severe, research on network traffic has become a hotspot in recent years. ${ }^{17-23}$

Some methods have been proposed to improve the performance of network traffic, such as modifying the underlying network structure, developing better routing strategies, or designing a better assignment of capacity distribution.

In the case of modifying the underlying network structure, Liu et al. ${ }^{17}$ proposed a novel strategy to enhance handling capacity by closing or cutting some links among some high-degree nodes. Similarly, a method was introduced in Ref. 18 to enhance transport efficiency by kicking out the edges linking to nodes with large betweenness. Huang et al. $\frac{19}{}$ proved that homogeneous networks can support a large critical packet generation rate under the congestion-free state. Modifying the underlying network topology is also called "hard" strategy in a recent review. 20

Due to the high cost of "hard" strategy, "soft" strategy, which develops a better routing strategy, is comparatively preferable. An efficient routing strategy, which redistributes the heavy load on central nodes to peripheral nodes, was proposed by Yan et al. ${ }^{6}$ Based on the global information of queue length of nodes, Ling et al. $\stackrel{7}{7}$ proposed a global dynamic routing strategy, significantly improving the network capacity. A routing algorithm was introduced to combine static structural properties and dynamic traffic conditions together in Ref. 21, where network capacity was enlarged considerably. When network size is large, the global information is rarely available. So a routing strategy based on local structural information was introduced by Wang et al. ${ }^{8}$

Another method is to optimize the allocation of the limited capacity resources, such as node's delivery capacity and band of links. Yang et al. ${ }^{22}$ found that when delivering capacity of a node is proportional to its degree with an exponent, transportation efficiency can be greatly improved. An optimal resource allocation strategy was proposed by Ling et al. ${ }^{23}$ where the network resource is allocated to each node according to its betweenness. Liu et al. ${ }^{24}$ studied the resource allocation from a more comprehensive perspective where both allocating the capacities of networks and distributing the rates of traffic flows are considered. They built an optimization problem to optimize the resource allocation and maximize the network utility at the same time.

In most previous works, first-in-first-out (FIFO) queuing strategy is widely used. It is true that FIFO queuing strategy is a simple and straight strategy, but it is not an optimal strategy. The pre-set packet priority is introduced by Kim et al. ${ }^{25}$ which means that in congestion state, packets with priority will be delivered first. It is showed that traffic is improved in the congested region under the priority queuing protocol, and it is worsened in the free-flow region. Inspired by this work, Du et al. ${ }^{26}$ proposed a shortest-remaining-path-first (SRPF) queue strategy, which determines a packet's priority according to the distance between its current location and destination, namely remaining-path-length. It is found that the transportation efficiency 
is significantly improved. However, SRPF strategy only considers remaining-pathlength, neglecting dynamic information of traffic load. When a packet $m$ arrives at node $i$, the packet's delivery priority is unable to update its value according to traffic congestion state. In this paper, we propose a hybrid queuing strategy (HQS), which combines the remaining-path-length and local dynamic information. Compared with the strategy based on the exclusive remaining-path-length, the traffic efficiency of networks utilizing HQS is further enhanced.

The paper is organized as follows. In Sec. 2, we describe the traffic model and the HQS in detail. In Sec. 3, the simulation results and discussions are provided. The work is concluded in the last section.

\section{The Model}

Since many traffic systems, such as air transportation network, ${ }^{27,28}$ follow powerlaw degree distribution, the well-known Barabási-Albert (BA) scale-free network model $^{17}$ is adopted to represent the physical infrastructure of traffic system in this paper. Growth and preferential attachment are two main properties of BA scale-free network. The BA network model starts from a fully connected graph with $m_{0}$ nodes and at each time step, a new node is linked to $m$ existing nodes according to the preferential attachment rule. Preferential attachment means that the probability for a new node connecting to an existing node $i$ is proportional to the degree of node $i$. In this paper, we set the network parameter as $N=500$ and $m_{0}=m=2$, where $N$ is the network size. All the nodes in the network are equally considered as hosts and the routers.

The network traffic model is described as follows. At each time step, there are $R$ packets generated with randomly chosen sources and destinations. Packets are delivered according to the shortest path strategy. Each node knows the queue length of its nearest-neighboring. Once a packet arrives at its destination, the packet will be removed from the system. One node can deliver at most $C=1$ packets in one time step. (We also checked the simulation results when $C$ is greater than 1 , and found that the simulation results are similar as the result of $C=1$.) The queue buffer of each node is unlimited.

If the node delivery capacity is larger than the queue length, all packets can be delivered. Contrarily, if the queue length is larger than node delivery, $C$ packets are picked to be delivered according to the queuing strategy and others have to wait in the buffer. From all the packets waiting at node $i$, we pick $C$ packets to deliver according to the HQS. Here, we define a parameter

$$
L_{m}=h^{*} d_{m}+(1-h)^{*} Q_{m},
$$

where $d_{m}$ is the remaining-path-length of packet $m, Q_{m}$ is the number of competitive packets in next arrival node of packet $m$. Competitive packets for packet $m$ refer to packets whose remaining-path-length is not larger than that of packet $m$. Packets will be delivered according to $L_{m}$ in ascending order. Parameter $h$ is used to 
adjust balance between the remaining-path-length and queue length of competitive packets in next arrival node. When $h=1$, our HQS equals to SRPF strategy. When $h=0$, the HQS ignores the packets' remaining-path-length and only considers local dynamic information.

\section{Simulation Results and Discussion}

Firstly, the order parameter proposed by Arenas et al. ${ }^{29}$ is adopted to characterize the transport efficiency:

$$
\eta=\frac{1}{R} \lim _{t \rightarrow \infty} \frac{\left\langle\Delta N_{P}\right\rangle}{\Delta t},
$$

where $\langle\cdots\rangle$ indicates average in time windows $\Delta t, \Delta N_{P}=N_{P}(t+\Delta t)-N_{P}(t)$ and $N_{P}(t)$ is the total number of packets in network at time step $t$. When $\eta \approx 0$, the system is in free-flow state. When $\eta>0$, some packets are accumulated in the system and the system gets into congestion state. There exists a critical value $R_{c}$ where a phase transition occurs from free-flow state to congestion. When $R<R_{c}$, created and removed packets are balanced, and $\eta \approx 0$. If $R>R_{c}$, packets pile up within the network because of limited delivery capacity.

We compare the order parameter $\eta(R)$ of the different queuing strategies, FIFO, SRPF and HQS. From Fig. 1, we can see that the critical value $R_{c}$ exhibits no evident improvement under hybrid queuing strategy. From previous studies, we know that when delivery capacity of each nodes is fixed to $C=1$, critical value $R_{c}$ can be estimated as ${ }^{23,30}$

$$
R_{c}=N(N-1) / B_{\max },
$$

where $N$ is the network size and $B_{\max }$ is the largest algorithmic betweenness of the network. Queuing strategies do not change the value of $N, B_{\max }$, hence the network capacity $R_{c}$ remains unchanged. ${ }^{26}$

Queuing strategy cannot enlarge network capacity $R_{c}$, but good queuing strategy can improve transport efficiency, especially in the congestion state. From Fig. 1, it can be observed that when $R$ becomes larger, the order value of FIFO is the largest, followed by SRPF, and the order value of HQS is the lowest. Lower value of $\eta(R)$ means less packets accumulated in the network and better transport efficiency.

Expect for order parameter $\eta(R)$, traffic efficiency can also be measured by packet arrival rate $Q$, which is defined $\mathrm{as}^{9,26}$

$$
Q=N_{\text {arrive }} / N_{\text {total }}
$$

where $N_{\text {arrive }}$ is the number of arrived packets and $N_{\text {total }}$ is the number of all generated packets during the whole transport process. Figure 2 shows the relationship between $Q$ (packets arrival rate) and $R$. When $R$ is small, all packets can arrive at their destination successfully, thus $Q=1$. When $R$ becomes larger, the value of $Q$ drops. We can see from Fig. 2 that the $Q$ of FIFO is the lowest, followed by SRPF, 




Fig. 1. (Color online) The relationship between the order parameter $\eta(R)$ and the packet generate rate $R$ under three different queuing strategies. The inset is the relationship between the order parameter $\eta(R)$ and the tunable parameter $h$ with packet generate rate $R=20$.

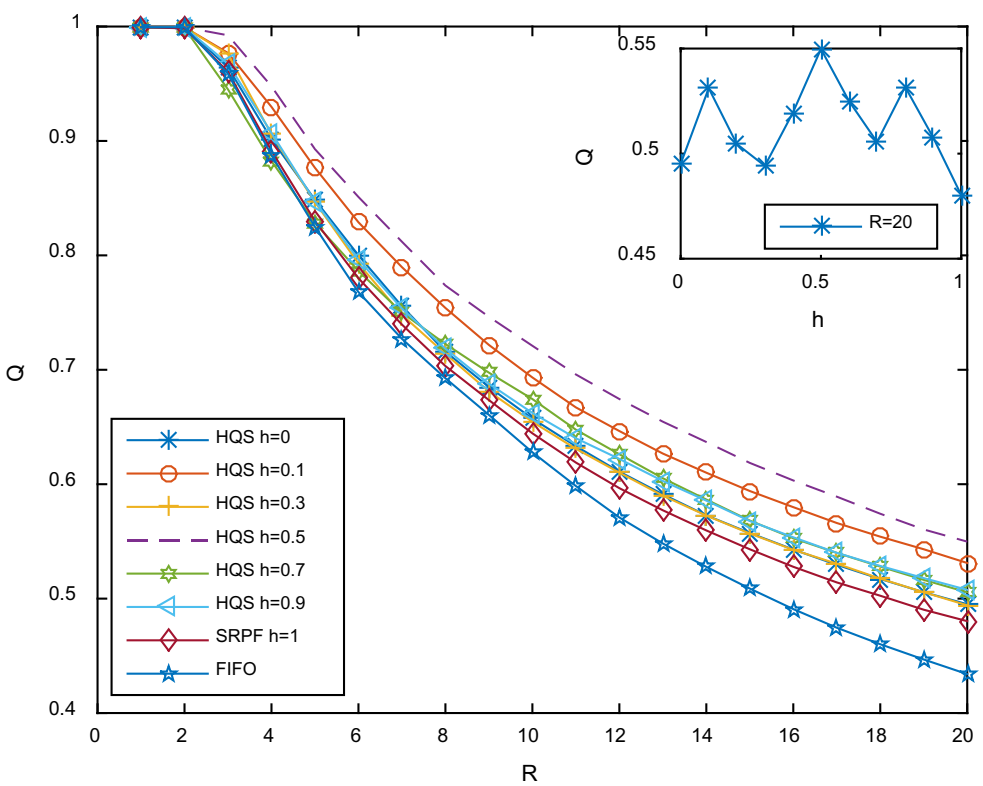

Fig. 2. (Color online) The relationship between the arrival rate $Q$ and the packet generate rate $R$ under different queuing strategies. The inset is the relationship between the arrival rate $Q$ and the tunable parameter $h$ with packet generate rate $R=20$. 
and HQS can achieve the highest value of $Q$. The higher value of $Q$ means higher transport efficiency, because more packets can arrive at the destination successfully.

Different values of $h$ mean different balances between the remaining-path-length and queue length of competitive packets in next arrival node. When $h=1$, the HQS equals SRPF strategy which ignores the queue length of competitive packets in next arrival node. Packets' delivery priority cannot be modified according to the network congestion state. Each node prefers to deliver packets with the shortest remaining-path-length, which inevitably leads to the hub nodes congestion. When $h=0$, the hybrid queue strategy ignores the packets' remaining-path-length, which means once there are some packets accumulated in the hub nodes, other nodes will prefer to deliver long length packet which do not pass hub nodes. When value of $h$ is between 0 and 1, the hybrid queue strategy keeps balance between the remaining-path-length and the queue length of competitive packets in next arrival nodes. From subgraphs of Figs. 1 and 2, we can see when $R=20$, the lowest order parameter and highest arrive rate correspond to $h=0.5$. Extensive experiment results demonstrate that the optimal value of $h$ changes with packet generate rate and network size.

We also compare average traveling time of arrived packets $\langle T\rangle$ under three different queuing strategies. For simplicity, only the simulation result of HQS with $h=0.5$ is exhibited. From Fig. 3(a), we can see that when $R$ is small, the network

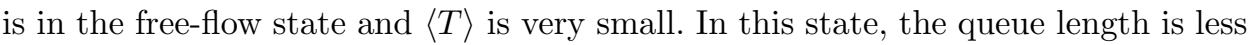
than the node deliver capacity, so all packets can be delivered and using different queuing strategies makes no differences. When $R$ becomes larger, the network is in the congestion state and $\langle T\rangle$ increases rapidly. In the congestion state, the queue length is large but the delivery capacity is limited, therefore we need to pick some packets to be delivered first. Modifying queuing strategy is actually to find a better way to use the limited delivery capacity in congestion state. We can find from Fig. 3(a) that the $\langle T\rangle$ of HQS is much smaller than that of FIFO and SRPF strategies in congestion state, which validates the enhanced performance of our hybrid strategy.

Figure 3(b) depicts different load distribution of three queuing strategies with $R=20$. The load is defined as the queue length of each node after 5000 time steps. Under HQS, hub nodes with large degree have less burden than SRPF and FIFO strategy. In SRPF strategy, packets that will pass through hub nodes are more likely to be chosen because of short remaining-path-length, which will lead to heavy burden of central node and is harmful to traffic efficiency. We overcome this shortcoming by taking local dynamic information into consideration. When the queue length of hub nodes becomes too large, other nodes will deliver less packet to the hub nodes, which alleviates the congestion on the hub nodes.

Figure 4 shows packet arrival rate for different path length packets. We can see that regardless of the adopted queuing strategy, short-path packets have higher arrival rate than long-path packets because they are nearer to the destination. 


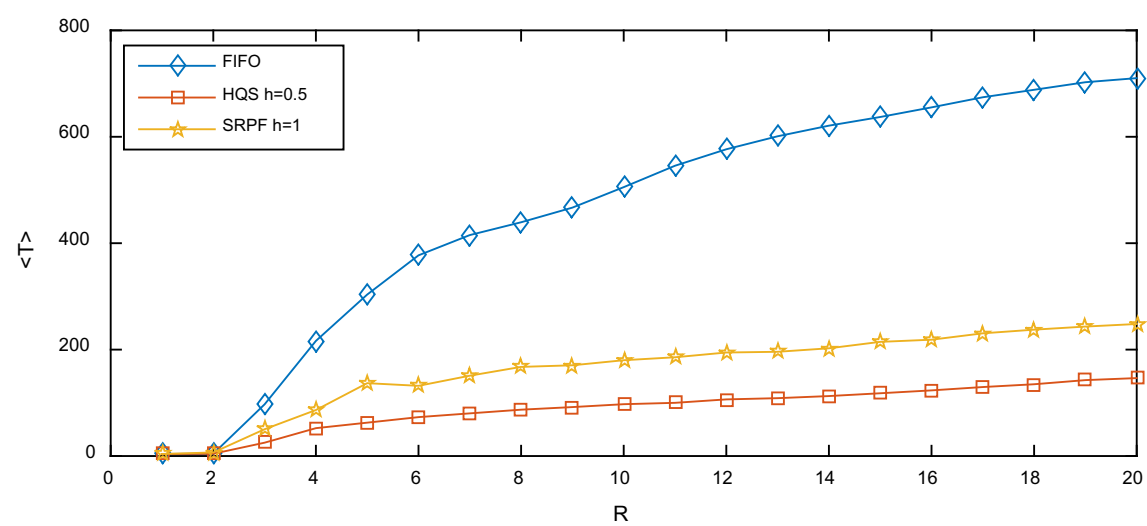

(a)

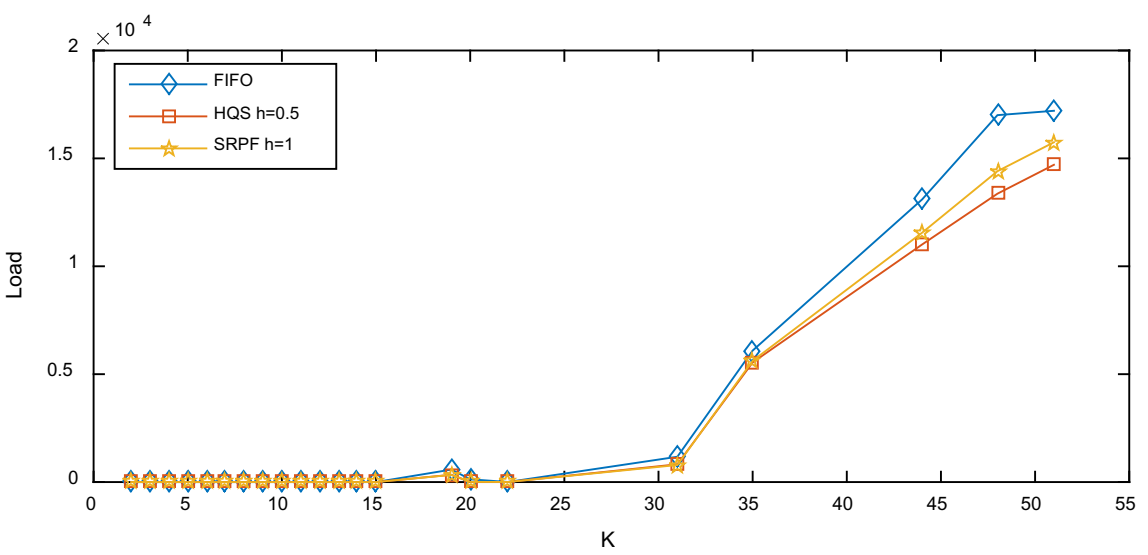

(b)

Fig. 3. (Color online) (a) The relationship of average travel time $\langle T\rangle$ and packet generate rate $R$ under different queue strategies. (b) The load distribution when congestion occurs for a BA network with $R=20$. Load means the queue length of nodes and $K$ means the degree of node.

However, the FIFO strategy shows no prejudice for long-path packets. Under the FIFO queuing strategy, for packets with path length being larger than four, the arrival rate fluctuates around 30\%. In contrast, under SRPF strategy, the arrival rate monotonously decreases with path length. For packets where path length is no larger than four, the arrival rate under the SRPF strategy is higher than that of the FIFO strategy. But for long-path packets (path length is larger than four), the arrival rate under SRPF strategy is lower than that of FIFO strategy. The SRPF strategy improves the network efficiency while sacrificing long-path packets' benefit, since most packets are short-path packets (from the subgraph of Fig. 4, we can see that over $80 \%$ packets are $L \leq 4$ ). Figure 4 shows that the arrival rate under our HQS also monotonously decreases with path length, and the HQS also sacrifices long-path packets' benefit, but the cost is less compared to the SRPF strategy. 




Fig. 4. (Color online) Arrival rate for different length path packets under three different queuing strategy with $R=20$. The inset is cumulative distribution of path length for all packets, with $R=20$.

When $h \neq 1$, our HQS takes the local dynamic into consideration. When hub nodes get into congestion state, high priority is given to long-path-length packets which will not pass through hub nodes. The arrival rate of long-path packets under HQS is larger than that of SRPF strategy. HQS achieves better network efficiency performance with a less cost compared to SRPF strategy.

\section{Conclusion}

In summary, we proposed a HQS on BA scale-free networks using the shortest path routing protocol. Under the HQS, packets' delivery priority was based on both the remaining-path-length and the queue length of next arrival node. A tunable parameter $h$ was introduced to adjust the balance between remaining-path-length and the competing queue length of next arrival node. Compared to the FIFO and the SRPF strategy, our method can further enhance the transport efficiency, such as the average traveling time and packets arrive rate, in congestion situation.

\section{Acknowledgments}

This work is supported by the National Natural Science Foundation for Young Scientists of China (Grant No. 61401011) and National Sci-Tech Support Program (Grant No. 2015BAG15B01). 


\section{References}

1. A. E. Motter and Y.-C. Lai, Phys. Rev. E 66 (2002) 065102.

2. S. V. Buldyrev, R. Parshani, G. Paul, H. E. Stanley and S. Havlin, Nature 464 (2010) 08932.

3. Y.-X. Xia, J. Fan and D. Hill, Physica A 389 (2010) 1281.

4. M. Perc and A. Szolnoki, BioSystems 99 (2010) 109.

5. W.-B. Du, X.-B. Cao, M.-B. Hu and W.-X. Wang, Europhys. Lett. 87 (2009) 60004.

6. G. Yan, T. Zhou, B. Hu, Z.-Q. Fu and B.-H. Wang, Phys. Rev. E 73 (2006) 046108.

7. X. Ling, M.-B. Hu, R. Jiang and Q.-S. Wu, Phys. Rev. E 81 (2010) 016113.

8. W.-X. Wang, B.-H. Wang, C.-Y. Yin, Y.-B. Xie and T. Zhou, Phys. Rev. E 73 (2006) 026111.

9. C. Hong, Physica A 424 (2015) 242.

10. Y. Deng, M. Sankaran and D.-Y. Zhou, Int. J. Unconv. Comput. 11 (2015) 227.

11. W. Jiang, B.-Y. Wei, J. Zhan, C.-H. Xie and D.-Y. Zhou, Comput. Ind. Eng. 101 (2016) 260.

12. W.-B. Du, Y. Gao, C. Liu and Z. Zheng, Appl. Math. Comput. 268 (2015) 832.

13. W.-B. Du, W. Ying, G. Yan, Y.-B. Zhu and X.-B. Cao, IEEE T. Circuits-II (in press), doi:10.1109/TCSII.2016.2595597.

14. F. Tan, J.-J. Wu, Y.-X. Xia and C.-K. Tse, Phys. Rev. E 89 (2014) 062813.

15. D. J. Watts and S. H. Strogatz, Nature 393 (1998) 440.

16. A. L. Barabási and R. Albert, Science 286 (1999) 509.

17. Z. Liu, M.-B. Hu, R. Jiang, W.-X. Wang and Q.-S. Wu, Phys. Rev. E 76 (2007) 037101.

18. G.-Q. Zhang, D. Wang and G.-J. Li, Phys. Rev. E 76 (2007) 017101.

19. W. Huang and T. W. S. Chow, Physica A 389 (2010) 871.

20. S.-Y. Chen, W. Huang, C. Cattani and G. Altieri, Math. Probl. Eng. 2012 (2012) 732698.

21. F. Tan and Y.-X. Xia, Physica A 392 (2013) 4146.

22. H.-X. Yang, W.-X. Wang, Z.-X. Wu and B.-H. Wang, Physica A 387 (2008) 6857.

23. X. Ling, M.-B. Hu and J.-C. Long, Chin. Phys. B 22 (2013) 018904.

24. H.-Y. Liu and Y.-X. Xia, IEEE Trans. Circuits Syst. II. Exp. Briefs 62 (2015) 706.

25. K. Kim, B. Kahng and D. Kim, Eur. Phys. Lett. 86 (2009) 58002.

26. W.-B. Du, Z.-X. Wu and K.-Q. Cai, Physica A 392 (2013) 3505.

27. W.-B. Du, X.-L. Zhou, O. Lordan, Z. Wang, Z. Chen and Y.-B. Zhu, Transport. Res. E 89 (2016) 108.

28. W.-B. Du, B.-Y. Liang, G. Yan, O. Lordan and X.-B. Cao, Chinese J. Aeronaut. (in press), arXiv:1608.00142.

29. A. Arenas, A. Díaz-Guilera and R. Guimerà, Phys. Rev. Lett. 86 (2001) 3196.

30. M. E. J. Newman, Phys. Rev. E 64 (2001) 016132. 